Appl. Phys. B 58, 123-132 (1994)

\title{
Experimental Verification of a Zero-Dimensional Model of the Ionization Kinetics of $\mathrm{XeCl}$ Discharges
}

\author{
H. Lück, D. Loffhagen, W. Bötticher \\ Institut für Plasmaphysik, Universität Hannover, Callinstrasse 38, D-30167 Hannover, Germany (Fax: +49-511/762-2784)
}

Received 7 July 1993/Accepted 21 October 1993

\begin{abstract}
An improved 0-dimensional model for $\mathrm{XeCl}$ high-pressure glow discharges is presented. Calculated discharge voltages are compared with precise measurements at a small, very homogeneous discharge. Excellent agreement in a wide parameter field demonstrates that this model may serve as a reference for simpler models describing the ionization kinetics.
\end{abstract}

PACS: $42.55 . \mathrm{Gp}, 52.80,82.20$

Models of Rare-Gas Halide (RGH) excimer lasers have been developed for more than a decade. So far no final agreement on the reaction kinetics and the treatment of the electron kinetics could be reached. Presently the most reliable approach to understand the kinetics of $\mathrm{RGH}$ excimer lasers is the comparison of spatially homogeneous (0-dimensional) model calculations with experimental data of homogeneous RGH discharges. Consistent two- or three-dimensional models including the full reaction kinetics as well as the nonMaxwellian electron energy distribution function and the space-charge-generated electrical fields do not exist. Due to numerical problems, the uncertainties of the quantitative aspects of the reaction kinetics, and the large computing times to be expected, current multidimensional models [1-6] use a simplified treatment of the reaction kinetics based on the results of 0 -dimensional models. Such simplifications typically comprise a reduction of the number of particle components of the discharge plasma and their collision processes, the renunciation of the solution of the electron Boltzmann equation for the determination of the electron kinetics, and the use of rate coefficients that are either constants or functions of the electron temperature or the reduced electrical field.

In order to check the predictions of 0-dimensional models precisely we developed a configuration for the generation of small homogeneous high-pressure glow discharges [7]. In that way we avoided the inaccuracies of experimental data gained from discharges in laser devices. In this paper we present the theoretical model of this discharge configuration and discuss in detail the comparison with experimen- tal results. The model calculations are performed with our self-consistent 0-dimensional model PINBED [8]. In contrast to most 0 -dimensional models that use a simplified quasi-stationary treatment of the electron kinetics discussed in detail in [9], our model uses a nonstationary treatment of the electron Boltzmann equation including superelastic collisions and the electron-electron interaction. This nonstationary electron kinetic equation is solved with a very efficient iterative technique allowing a high energy resolution with typically 1000 and more grid points. This fine energy grid accurately describes the fine structure of the electron impact cross sections of several species involved in excimer-laser discharges [10], whereas recent models including a nonstationary electron kinetics $[11,12]$ have got an energy resolution of the order of about 100 grid points only.

The extensive reaction kinetic model used for our model calculations of $\mathrm{Ne} / \mathrm{Xe} / \mathrm{HCl}$ mixtures is based on the model of $[13,14]$, developed for investigations of $\mathrm{XeCl}$ lasers, and is updated taking recent literature into account. A detailed description of the full set of reactions allowing comparisons with various spectroscopically measured particle number densities is given with the main attention focussed on the ionization and recombination kinetics.

The results of the model calculations are compared with precise measurements of discharge voltage and current over a wide range of parameter variations. Comparative investigations of the reaction kinetic model with spectroscopic measurements of excited species number densities $\mathrm{Xe}^{*}, \mathrm{Cl}^{*}$, $\mathrm{H}^{*}, \mathrm{Ne}^{*}$, and $\mathrm{XeCl}^{*}$ are not discussed in this paper and will be presented in a subsequent paper [15].

\section{Description of the Experimental Setup}

As a detailed description of the experimental setup is given in [7] we will only give a short summary here. The experimental setup consists of a discharge chamber, an impulse generator to produce a well-defined voltage and current pulse, and an X-ray tube to provide sufficient preionization. The discharge chamber, mainly made of nickel-plated brass and PTFE, is capable of withstanding pressures of up 
Table 1. Standard discharge parameters

\begin{tabular}{ll}
\hline Discharge parameter & Value \\
\hline Gas mixture & $5: 75: 2920 \mathrm{mbar} \mathrm{HCl}: \mathrm{Xe}: \mathrm{Ne}$ \\
Applied voltage & $25.9 \mathrm{kV}$ \\
Current density & $\mathrm{ca} .250 \mathrm{~A} / \mathrm{cm}^{2}$ \\
Electrode gap & $18.9 \mathrm{~mm}$ \\
Preionization electron density & $1.2 \times 10^{9} / \mathrm{cm}^{3}$ \\
Length of voltage pulse & $100 \mathrm{~ns}$ \\
Discharge cross section & circular, diameter $=1.12 \mathrm{~cm}$ \\
Gas temperature & room temperature $=293 \pm 5 \mathrm{~K}$ \\
\hline
\end{tabular}

to 10 bars. Typical discharge parameters are given in Table 1 . An integrated capacitive voltage divider and a current viewing resistor allow precise measurements of the discharge voltage and current. A discussion of the accuracy of these measurements is given in [7]. The pulse generator with an output impedance of $50 \Omega$ delivers a single high-voltage impulse with a width of $100 \mathrm{~ns}$ and a variable charging voltage $U_{\mathrm{Ch}}$ ranging from $10 \mathrm{kV}$ to $30 \mathrm{kV}$. A current limiting resistor of $50 \Omega$ in series to the discharge limits the current to $I=U_{\mathrm{Ch}} / 100 \Omega=(100-300 \mathrm{~A})$. A home-made X-ray tube with a pulse length of about $50 \mathrm{~ns}$ gives a homogeneous preionization electron density of $10^{9} / \mathrm{cm}^{3}$. This value has been measured in situ for the gas mixture used [16]. The discharge cross section is determined by a set of lead apertures between the X-ray tube and the discharge region. The measurements presented in this paper have been done with a circular aperture with a radius of $5 \mathrm{~mm}$ and a gap between the plane-parallel electrodes of $18.9 \mathrm{~mm}$.

Increasing the gas pressure in the discharge chamber pushes the cathode outward. The electrode gap thereby increases by $0.06 \mathrm{~mm} / \mathrm{bar}$ thus changing $C_{\mathrm{Dis}}$ slightly. The corresponding change of the dividing ratio of the capacitive voltage divider has been measured to be $0.6 \% / \mathrm{bar}$ and is as well taken into account when comparing the experimental data with the model calculations.

\subsection{Discharge Homogeneity}

The discharge homogeneity was checked by taking twodimensional pictures of the visible and the UV emission of the discharge using a gated (5 ns) MCP-intensified CCD camera. We have to consider two different types of inhomogeneities that have an influence on the discharge voltage: discharge constrictions and electrode sheaths.

- Cathode spots developing during the ignition phase (see Sect. 3.1) can cause a constriction of the discharge in the vicinity of these spots. The use of carefully polished and cleaned copper or nickel electrodes can avoid the appearance of cathode spots. Therefore the constriction of the discharge could be avoided for a limited number of discharges and thus its influence on the discharge voltage can be studied. Since the dependence of the discharge voltage on the current density is small, we found that the formation of the spots decreases the discharge voltage at standard discharge conditions at the end of the quasi-steady-state phase (see Sect. 3.1) only by about $300 \mathrm{~V}$. The comparison of the model and the experiment has only been done for homogeneous conditions of the bulk plasma.

- An electrode sheath could only be detected in the cathode region. During the ignition phase a bright layer of about $100 \mu \mathrm{m}$ width develops in front of the cathode. This layer is most likely caused by a region of enhanced electrical field at the cathode surface comparable to the cathode fall of lowpressure glow discharges. In low-pressure glow discharges the cathode-fall voltage of some $100 \mathrm{~V}$ may take a significant part of the overall discharge voltage as the electrical field in the positive column is typically very small (some $\mathrm{V} / \mathrm{cm}$ ). In this high-pressure glow discharge the reduced electrical field $E / N$ is about the same as in low-pressure glow discharges but due to the high pressure the voltage drop in the positive column (bulk plasma) is much higher than the cathode-fall voltage.

In order to assure the validity of the 0-dimensional assumption of the model, the cathode fall has either to be known to take a negligible part of the overall discharge voltage or it has to be measured and then included in the model as an additional term to the discharge voltage. In our case we found that during the quasi-steady-state phase the cathode-fall voltage only takes a negligible part of the discharge voltage and has therefore not been been taken into account in our model calculations.

\subsection{Cathode-Fall Voltage}

The extension of the bright luminious region in front of the cathode is only about $100 \mu \mathrm{m}$. We therefore assume the extension of the cathode fall region to be negligible in comparison with the electrode gap. The discharge voltage $U_{\text {Dis }}$ can then be written as a sum of the cathode fall voltage $U_{\mathrm{c}}$ and the voltage of the bulk plasma $U_{\mathrm{b}}=E_{\mathrm{b}} d$ according to $U_{\text {Dis }}(d)=U_{\mathrm{c}}+E_{\mathrm{b}} d$, where $E_{\mathrm{b}}$ is the electrical field of the bulk plasma and $d$ the electrode gap. Keeping $E_{\mathrm{b}}$ constant, the cathode-fall voltage $U_{\mathrm{c}}=\left[d_{1} U_{\mathrm{Dis}}\left(d_{2}\right)-d_{2} U_{\mathrm{Dis}}\left(d_{1}\right)\right] /\left(d_{1}-\right.$ $d_{2}$ ) can be obtained by measuring the discharge voltages at two different electrode gaps $d_{1}$ and $d_{2}$.

It is essential that the other discharge parameters remain unchanged while changing the electrode gap. Thus, in order to keep $E_{\mathrm{b}}$ constant while changing the electrode gap, the applied voltage and the current-limiting resistor, accordingly, (to keep the current density constant) have to be changed.

Another way to obtain information about the cathode-fall voltage is to insert a thin electrically floating electrode between the cathode and the anode. If the thickness of the foil is negligible (e.g. $50 \mu \mathrm{m}$ ) in comparison with the electrode gap, we thereby add another cathode and anode fall to the discharge while keeping all the other discharge parameters constant. The use of an aluminium foil as an additional electrode changes the preionization electron density behind this electrode by less than $1 \%$. Pictures taken with this configuration show no discharge constrictions below or above the foil. Therefore, one has the sarne boundary condition as on the proper electrodes and an additional cathode and anode fall are inserted. If the anode fall voltage is much smaller than the cathode-fall voltage, the difference of the discharge 
voltages measured with and without the foil gives the cathode fall voltage. The virtual decrease of the electrode gap by inserting a conducting foil in-between the electrodes has an insignificant influence on the discharge voltage as the electrical field in the discharge is of the order of $2000 \mathrm{~V} / \mathrm{cm}$ (i.e. $10 \mathrm{~V} / 50 \mu \mathrm{m})$.

Whereas the former method requires the exact measurement of the electrode gap as well as of the discharge voltage, the latter one only requires exact data of the discharge voltage because the electrode gap remains unchanged. Therefore, the error of the latter method (about $300 \mathrm{~V}$ ) is smaller than that of the former one (about $600 \mathrm{~V}$ ). With both methods the measurements gave a cathode fall voltage of a few hundred volts at standard discharge conditions. A similar result has already been found by Cavenor and Meyer [17] for highpressure glow discharges in hydrogen. First model calculations for a $\mathrm{XeCl}$ discharge have been published by Belasri et al. [18]. The predicted cathode-fall voltage depends strongly on the secondary electron emission coefficient $\gamma$. Even if $\gamma=0.1$ one gets a value $(1300 \mathrm{~V})$ much larger than our measured cathode fall voltage.

Since the discharge current constricts faster when the electrode gap is shortened, a variation of the electrode gap can only give information about the cathode fall voltage during early discharge phases when the influence of the constriction on the discharge voltage is significantly less than the cathode fall voltage, i.e. in the first $20-30 \mathrm{~ns}$ of the quasi-steady-state phase (see Sect. 3.1).

\section{Description of the Discharge Model}

The self-consistent model of a preionized discharge-pumped excimer-laser plasma in the small volume discharge configuration consists of the electron Boltzmann equation for the determination of the electron kinetics, of the rate equation system of the various heavy particles occurring in the plasma, and of the equation system for the electrical circuit. Since this discharge configuration is not a laser device, the resonator equation of the photons in an optical cavity has not to be included. The approach of 0 -dimensional modelling is justified since the object of our experimental investigations is a very homogeneous glow discharge.

\subsection{Numerical Model}

The calculations are performed with the self-consistent model PINBED consisting of a repetition up to convergence for each time step of the successive solution of the coupled system of the rate equations for the heavy particles and the electrical circuit equations by means of the two-stage method of Steihaug and Wolfbrandt, and Scraton $[19,20]$ on the one side and of the nonstationary electron Boltzmann equation by means of a very efficient iterative technique on the other side. A detailed description of this code is given by Loffhagen and Winkler [8]. The solution of the rate and electrical circuit equations yields the temporal evolution of the heavy particle densities and the electrical field necessary for the determination of the electron kinetic quantities. The solution of the nonstationary electron Boltzmann equation provides the temporal evolution of the isotropic distribution function and thus of the macroscopic quantities electron density, mean electron energy, electron mobility, and the various rate coefficients for the electron-heavy-particle collision processes coupling the electron kinetics back to the heavy-particle kinetics and the electrical circuit.

The nonstationary treatment of the Boltzmann equation of the electrons is based on the conventional two-term approximation of the velocity distribution and a quasi-stationary description of the anisotropic part of the distribution. The validity of both approximations has been proved in [9]. The Boltzmann equation takes into account elastic collisions, excitation, dissociation, ionization, attachment, and recombination as well as second-kind collisions and electron-electron interaction. The electron energy grid is made up of 1000 equidistant intervals with the boundaries 0 and $U_{\infty}$. The upper energy limit $U_{\infty}$ is adjusted according to the instantaneous situation during the temporal evolution of the discharge. The initial distribution is taken to be a Maxwellian with a mean electron energy equivalent to the gas temperature of $293 \mathrm{~K}$.

Due to its modular structure the numerical model can easily be changed to different homogeneous discharge configurations and (within the validity limits of the nonstationary electron Boltzmann equation treatment) to other gas mixtures.

\subsection{Reaction Kinetic Model}

The full set of reactions used for our model calculations of $\mathrm{Ne} / \mathrm{Xe} / \mathrm{HCl}$ plasmas is reported in Table 2. It is based on the reaction kinetic model of $[13,14]$ and is updated taking recent literature into account. Thirty-seven heavy particle components $\left(\mathrm{Xe}, \mathrm{Xe}^{*}, \mathrm{Xe}^{* *}, \mathrm{Xe}^{+}, \mathrm{Xe}_{2}^{*}, \mathrm{Xe}_{2}^{+}, \mathrm{Ne}\right.$, $\mathrm{Ne}^{*}, \mathrm{Ne}^{* *}, \mathrm{Ne}^{+}, \mathrm{HCl}(v=0), \mathrm{HCl}(v=1), \mathrm{HCl}(v=2)$, $\mathrm{HCl}(v=3), \mathrm{HCl}(v=4), \mathrm{HCl}(v=5), \mathrm{HCl}^{*}(B, C), \mathrm{HCl}^{+}$, $\mathrm{Cl}, \mathrm{Cl}^{*}, \mathrm{Cl}^{* *}, \mathrm{Cl}^{+}, \mathrm{Cl}^{-}, \mathrm{Cl}_{2}^{*}, \mathrm{H}, \mathrm{H}^{*}(n=2), \mathrm{H}^{*}(n=3)$, $\mathrm{H}^{*}(n=4), \mathrm{H}^{*}(n=5), \mathrm{H}^{*}(n=6), \mathrm{H}^{*}(n=7), \mathrm{H}^{+}, \mathrm{H}^{-}$, $\left.\mathrm{XeCl}^{*}(B), \mathrm{XeCl}(X), \mathrm{Xe}_{2} \mathrm{Cl}^{*}, \mathrm{NeXe}^{+}\right)$and the electron are necessary to specify the reactions allowing comparisons with measurements of discharge voltage, discharge current, and various particle number densities. The number of reactions relevant to the solution of the nonstationary electron Boltzmann equation amounts to 22 elastic and 89 inelastic collision processes dealt with by collision cross sections as well as four electron-loss processes by recombination and four electron-production processes by detachment and ionization for which only rate coefficients are known. These reactions are listed at the beginning of Table 2 .

In this paper we concentrate on the comparison of measured and calculated discharge voltages and currents. The improvements of the ionization and recombination kinetics relevant to the determination of the plasma conductivity will be discussed in the following. Analogous to [14] the electronic manifold of atomic xenon and neon is represented by two excited states, defined by a combination of the individual $(n+1) s$ and $(n+1) s^{\prime}$ levels or the individual $(n+1) p$ and $(n+1) p^{\prime}$ levels with the statistical weights of 12 and 36 , respectively, where $n=5$ for xenon and $n=2$ for neon. It is expected that a more sophisticated model of the excited 
Table 2. Reactions, collision cross sections, and rate coefficients used for our model calculations. The middle column of the electron-collision cross section data gives the energy range in $\mathrm{eV}$ for which data of the given reference were used. All rate coefficients are in units of $\mathrm{cm}^{3} \mathrm{~s}^{-1}$, except that for reactions involving three species on the left-hand side the units are $\mathrm{cm}^{6} \mathrm{~s}^{-1}$. Rates for emission are given in units of $\mathrm{s}^{-1}$; $T_{\mathrm{e}}$ is the electron temperature in $\mathrm{eV}$

\begin{tabular}{|c|c|c|c|c|}
\hline Number & Process & Energy range & Reference & \\
\hline \multicolumn{5}{|c|}{ Elastic electron collisions } \\
\hline (1) & $\mathrm{e}+\mathrm{Ne} \rightarrow \mathrm{e}+\mathrm{Ne}$ & $\begin{array}{l}0-2.176 \\
5-200\end{array}$ & $\begin{array}{l}\text { O'Malley, Crompton } \\
\text { Fon, Berrington }\end{array}$ & $\begin{array}{l}{[21]} \\
{[22]}\end{array}$ \\
\hline (2) & $\mathrm{e}+\mathrm{Xe} \rightarrow \mathrm{e}+\mathrm{Xe}$ & $\begin{array}{l}0.01-20 \\
20-200\end{array}$ & $\begin{array}{l}\text { Sin Fai Lam } \\
\text { Hayashi }\end{array}$ & $\begin{array}{l}{[23]} \\
{[24]}\end{array}$ \\
\hline (3) & $\mathrm{e}+\mathrm{HCl}(0) \rightarrow \mathrm{e}+\mathrm{HCl}(0)$ & $\begin{array}{l}0.01-10 \\
10-200\end{array}$ & $\begin{array}{l}\text { Padia et al. } \\
\text { Hammer }\end{array}$ & $\begin{array}{l}{[25]} \\
{[26]}\end{array}$ \\
\hline (4) & $\mathrm{e}+\mathrm{Cl} \rightarrow \mathrm{e}+\mathrm{Cl}$ & $0-100$ & Rogoff et al. & [27] \\
\hline (5) & $\mathrm{e}+\mathrm{H} \rightarrow \mathrm{e}+\mathrm{H}$ & $\begin{array}{c}0-8.7 \\
11-50\end{array}$ & $\begin{array}{l}\text { Fon et al. } \\
\text { Fon et al. }\end{array}$ & [28] \\
\hline$(6)$ & $\mathrm{e}+\mathrm{Ne}^{*} \rightarrow \mathrm{e}+\mathrm{Ne}^{*}$ & $0-200$ & Analogous to (1) & \\
\hline (7) & $\mathrm{e}+\mathrm{Ne}^{* *} \rightarrow \mathrm{e}+\mathrm{Ne}^{* *}$ & $0-200$ & Analogous to (1) & \\
\hline (8) & $\mathrm{e}+\mathrm{Xe}^{*} \rightarrow \mathrm{e}+\mathrm{Xe}^{*}$ & $0.01-200$ & Analogous to (2) & \\
\hline (9) & $\mathrm{e}+\mathrm{Xe} \mathrm{e}^{* *} \rightarrow \mathrm{e}+\mathrm{Xe}^{* *}$ & $0.01-200$ & Analogous to (2) & \\
\hline$(10-14)$ & $\mathrm{e}+\mathrm{HCl}(v) \rightarrow \mathrm{e}+\mathrm{HCl}(v), 1 \leq v \leq 5$ & $0.01-200$ & Analogous to (3) & \\
\hline (15) & $\mathrm{e}+\mathrm{Cl}^{*} \rightarrow \mathrm{e}+\mathrm{Cl}^{*}$ & $0-100$ & Analogous to (4) & \\
\hline (16) & $\mathrm{e}+\mathrm{Cl}^{* *} \rightarrow \mathrm{e}+\mathrm{Cl}^{* *}$ & $0-100$ & Analogous to (4) & \\
\hline$(17-22)$ & $\mathrm{e}+\mathrm{H}^{*}(n) \rightarrow \mathrm{e}+\mathrm{H}^{*}(n), 2 \leq n \leq 7$ & $0-50$ & Analogous to (5) & \\
\hline \multicolumn{5}{|c|}{ Electron-collision excitation and superelastic collisions } \\
\hline$(23,24)$ & $\mathrm{Ne}+\mathrm{e} \leftrightarrow \mathrm{Ne}^{*}+\mathrm{e}$ & $16.6-100$ & Puech, Mizzi & [30] \\
\hline$(25,26)$ & $\mathrm{Ne}+\mathrm{e} \leftrightarrow \mathrm{Ne}^{* *}+\mathrm{e}$ & $18.38-100$ & Puech, Mizzi & [30] \\
\hline \multirow[t]{2}{*}{$(27,28)$} & $\mathrm{Ne}^{*}+\mathrm{e} \leftrightarrow \mathrm{Ne}^{* *}+\mathrm{e}$ & $1.76-145$ & Seaton & [31] \\
\hline & & & Hyman & [32] \\
\hline$(29,30)$ & $\mathrm{Xe}+\mathrm{e}+\mathrm{Xe}^{*}+\mathrm{e}$ & $8.32-100$ & Puech, Mizzi & [30] \\
\hline$(31,32)$ & $\mathrm{Xe}+\mathrm{e}+\mathrm{Xe}^{* *}+\mathrm{e}$ & $9.58-100$ & Puech, Mizzi & [30] \\
\hline \multirow[t]{2}{*}{$(33,34)$} & $\mathrm{Xe}^{*}+\mathrm{e}+\mathrm{Xe}^{* *}+\mathrm{e}$ & $1.14-136$ & Seaton & [31] \\
\hline & & & Hyman & [32] \\
\hline$(35,36)$ & $\mathrm{HCl}(0)+\mathrm{e} \leftrightarrow \mathrm{HCl}(1)+\mathrm{e}$ & $0.33-5$ & Domcke, Mündel & [33] \\
\hline$(37,38)$ & $\mathrm{HCl}(0)+\mathrm{e} \leftrightarrow \mathrm{HCl}(2)+\mathrm{e}$ & $0.67-5$ & Domcke, Mündel & [33] \\
\hline$(39,40)$ & $\mathrm{HCl}(0)+\mathrm{e} \leftrightarrow \mathrm{HCl}(3)+\mathrm{e}$ & $1.0-5$ & Domcke, Mündel & [33] \\
\hline$(41,42)$ & $\mathrm{HCl}(0)+\mathrm{e} \leftrightarrow \mathrm{HCl}(4)+\mathrm{e}$ & $1.31-5$ & Domcke & [34] \\
\hline$(43,44)$ & $\mathrm{HCl}(0)+\mathrm{e} \leftrightarrow \mathrm{HCl}(5)+\mathrm{e}$ & $1.61-5$ & Domcke & [34] \\
\hline$(45)$ & $\mathrm{HCl}(0)+\mathrm{e} \leftrightarrow \mathrm{HCl}^{*}(A)+\mathrm{e}$ & $5.5-11$ & Davies & [35] \\
\hline$(46)$ & $\mathrm{HCl}(0)+\mathrm{e} \leftrightarrow \mathrm{HCl}^{*}(B, C)+\mathrm{e}$ & $9.3-100$ & Davies & [35] \\
\hline$(47,48)$ & $\mathrm{HCl}(1)+\mathrm{e} \leftrightarrow \mathrm{HCl}(2)+\mathrm{e}$ & $0.31-5$ & Domcke & [34] \\
\hline$(49,50)$ & $\mathrm{HCl}(1)+\mathrm{e} \leftrightarrow \mathrm{HCl}(3)+\mathrm{e}$ & $0.64-5$ & Domcke & [34] \\
\hline$(51,52)$ & $\mathrm{HCl}(1)+\mathrm{e} \leftrightarrow \mathrm{HCl}(4)+\mathrm{e}$ & $0.95-5$ & Domcke & [34] \\
\hline$(53,54)$ & $\mathrm{HCl}(1)+\mathrm{e} \leftrightarrow \mathrm{HCl}(5)+\mathrm{e}$ & $1.25-5$ & Domcke & [34] \\
\hline$(55,56)$ & $\mathrm{HCl}(2)+\mathrm{e} \leftrightarrow \mathrm{HCl}(3)+\mathrm{e}$ & $0.30-5$ & Domcke & [34] \\
\hline$(57,58)$ & $\mathrm{HCl}(2)+\mathrm{e} \leftrightarrow \mathrm{HCl}(4)+\mathrm{e}$ & $0.61-5$ & Domcke & [34] \\
\hline$(59,60)$ & $\mathrm{HCl}(2)+\mathrm{e} \leftrightarrow \mathrm{HCl}(5)+\mathrm{e}$ & $0.91-5$ & Domcke & [34] \\
\hline$(61,62)$ & $\mathrm{HCl}(3)+\mathrm{e} \leftrightarrow \mathrm{HCl}(4)+\mathrm{e}$ & $0.33-5$ & Analogous to $(35,36)$ & \\
\hline$(63,64)$ & $\mathrm{HCl}(3)+\mathrm{e} \leftrightarrow \mathrm{HCl}(5)+\mathrm{e}$ & $0.67-5$ & Analogous to $(37,38)$ & \\
\hline$(65,66)$ & $\mathrm{HCl}(4)+\mathrm{e} \leftrightarrow \mathrm{HCl}(5)+\mathrm{e}$ & $0.33-5$ & Analogous to $(35,36)$ & \\
\hline$(67,68)$ & $\mathrm{Cl}+\mathrm{e} \leftrightarrow \mathrm{Cl}^{*}+\mathrm{e}$ & $8.92-100$ & Ganas & [36] \\
\hline$(69,70)$ & $\mathrm{Cl}+\mathrm{e} \leftrightarrow \mathrm{Cl}^{* *}+\mathrm{e}$ & $10.28-100$ & Ganas & [36] \\
\hline$(71,72)$ & $\mathrm{Cl}^{*}+\mathrm{e} \leftrightarrow \mathrm{Cl}^{* *}+\mathrm{e}$ & $1.0-136$ & Estimated & \\
\hline \multirow[t]{2}{*}{$(73,74)$} & $\mathrm{H}+\mathrm{e} \leftrightarrow \mathrm{H}^{*}(2)+\mathrm{e}$ & $10.2-12.1$ & Callaway & [37] \\
\hline & & $12.2-54$ & Callaway & [38] \\
\hline$(75,76)$ & $\mathrm{H}+\mathrm{e} \leftrightarrow \mathrm{H}^{*}(3)+\mathrm{e}$ & $12.1-100$ & Callaway et al. & [39] \\
\hline$(77,78)$ & $\mathrm{H}^{*}(2)+\mathrm{e} \leftrightarrow \mathrm{H}^{*}(3)+\mathrm{e}$ & $1.89-100$ & Callaway et al. & [39] \\
\hline$(79,80)$ & $\mathrm{H}^{*}(3)+\mathrm{e} \leftrightarrow \mathrm{H}^{*}(4)+\mathrm{e}$ & $0.66-50$ & Vriens, Smeets & [40] \\
\hline$(81,82)$ & $\mathrm{H}^{*}(4)+\mathrm{e} \leftrightarrow \mathrm{H}^{*}(5)+\mathrm{e}$ & $0.3-50$ & Vriens, Smeets & [40] \\
\hline$(83,84)$ & $\mathrm{H}^{*}(5)+\mathrm{e} \leftrightarrow \mathrm{H}^{*}(6)+\mathrm{e}$ & $0.16-50$ & Vriens, Smeets & {$[40]$} \\
\hline$(85,86)$ & $\mathrm{H}^{*}(6)+\mathrm{e} \leftrightarrow \mathrm{H}^{*}(7)+\mathrm{e}$ & $0.1-50$ & Vriens, Smeets & [40] \\
\hline \multicolumn{5}{|c|}{ Electron-collision ionization and detachment } \\
\hline$(87)$ & $\mathrm{Ne}+\mathrm{e} \rightarrow \mathrm{Ne}^{+}+\mathrm{e}+\mathrm{e}$ & $21.6-200$ & Wetzel et al. & [41] \\
\hline$(88)$ & $\mathrm{Ne}^{*}+\mathrm{e} \rightarrow \mathrm{Ne}^{+}+\mathrm{e}+\mathrm{e}$ & $4.94-100$ & Hyman & [42] \\
\hline (89) & $\mathrm{Ne}^{* *}+\mathrm{e} \rightarrow \mathrm{Ne}^{+}+\mathrm{e}+\mathrm{e}$ & $3.18-50$ & Hyman & [42] \\
\hline$(90)$ & $\mathrm{Xe}+\mathrm{e} \rightarrow \mathrm{Xe}^{+}+\mathrm{e}+\mathrm{e}$ & $12.1-140$ & Wetzel et al. & [41] \\
\hline (91) & $\mathrm{Xe}^{*}+\mathrm{e} \rightarrow \mathrm{Xe}^{+}+\mathrm{e}+\mathrm{e}$ & $3.81-100$ & Hyman & [42] \\
\hline
\end{tabular}


Table 2 (continued)

\begin{tabular}{|c|c|c|c|c|}
\hline Number & Process & Rate coefficient & References & \\
\hline$(92)$ & $\mathrm{Xe}^{* *}+\mathrm{e} \rightarrow \mathrm{Xe}^{+}+\mathrm{e}+\mathrm{e}$ & $2.39-100$ & Hyman & [42] \\
\hline (93) & $\mathrm{HCl}(0)+\mathrm{e} \rightarrow \mathrm{HCl}^{+}+\mathrm{e}+\mathrm{e}$ & $12.74-100$ & Davies & [35] \\
\hline (94) & $\mathrm{Cl}+\mathrm{e} \rightarrow \mathrm{Cl}^{+}+\mathrm{e}+\mathrm{e}$ & $13-100$ & Hayes et al. & [43] \\
\hline (95) & $\mathrm{Cl}^{*}+\mathrm{e} \leftrightarrow \mathrm{Cl}^{+}+\mathrm{e}+\mathrm{e}$ & $4.09-107$ & Estimated & \\
\hline (96) & $\mathrm{Cl}^{* *}+\mathrm{e} \leftrightarrow \mathrm{Cl}^{+}+\mathrm{e}+\mathrm{e}$ & $2.73-107$ & Estimated & \\
\hline (97) & $\mathrm{H}+\mathrm{e} \leftrightarrow \mathrm{H}^{+}+\mathrm{e}+\mathrm{e}$ & $13.6-198$ & Shah et al. & [44] \\
\hline (98) & $\mathrm{H}^{*}(2)+\mathrm{e} \rightarrow \mathrm{H}^{+}+\mathrm{e}+\mathrm{e}$ & $3.4-50$ & Vriens, Smeets & [40] \\
\hline (99) & $\mathrm{H}^{*}(3)+\mathrm{e} \rightarrow \mathrm{H}^{+}+\mathrm{e}+\mathrm{e}$ & $1.5-50$ & Vriens, Smeets & {$[40]$} \\
\hline$(100)$ & $\mathrm{H}^{*}(4)+\mathrm{e} \rightarrow \mathrm{H}^{+}+\mathrm{e}+\mathrm{e}$ & $0.85-50$ & Vriens, Smeets & [40] \\
\hline$(101)$ & $\mathrm{H}^{*}(5)+\mathrm{e} \rightarrow \mathrm{H}^{+}+\mathrm{e}+\mathrm{e}$ & $0.54-50$ & Vriens, Smeets & [40] \\
\hline$(102)$ & $\mathrm{H}^{*}(6)+\mathrm{e} \rightarrow \mathrm{H}^{+}+\mathrm{e}+\mathrm{e}$ & $0.37-50$ & Vriens, Smeets & [40] \\
\hline (103) & $\mathrm{H}^{*}(7)+\mathrm{e} \rightarrow \mathrm{H}^{+}+\mathrm{e}+\mathrm{e}$ & $0.27-50$ & Vriens, Smeets & [40] \\
\hline (104) & $\mathrm{Cl}^{-}+\mathrm{e} \rightarrow \mathrm{Cl}+\mathrm{e}+\mathrm{e}$ & $3.61-100$ & Massey & [45] \\
\hline \multicolumn{5}{|c|}{ Dissociative electron attachment } \\
\hline$(105)$ & $\mathrm{HCl}(0)+\mathrm{e} \rightarrow \mathrm{H}+\mathrm{Cl}^{-}$ & $0.8-2.5$ & Domcke, Mündel & [33] \\
\hline$(106)$ & $\mathrm{HCl}(0)+\mathrm{e} \rightarrow \mathrm{H}^{-}+\mathrm{Cl}$ & $5.6-11$ & Davies & [35] \\
\hline$(107)$ & $\mathrm{HCl}(1)+\mathrm{e} \rightarrow \mathrm{H}+\mathrm{Cl}^{-}$ & $0.45-2.5$ & Domcke, Mündel & [33] \\
\hline (108) & $\mathrm{HCl}(2)+\mathrm{e} \rightarrow \mathrm{H}+\mathrm{Cl}^{-}$ & $0.1-2.5$ & Domcke, Mündel & [33] \\
\hline$(109)$ & $\mathrm{HCl}(3)+\mathrm{e} \rightarrow \mathrm{H}+\mathrm{Cl}^{-}$ & $0-0.4$ & Bardsley, Wahedra & {$[46]$} \\
\hline & & $0.4-2.5$ & Analogous to $(108)$ & \\
\hline$(110)$ & $\mathrm{HCl}(4)+\mathrm{e} \rightarrow \mathrm{H}+\mathrm{Cl}^{--}$ & $0-2.5$ & Analogous to (109) & \\
\hline (111) & $\mathrm{HCl}(5)+\mathrm{e} \rightarrow \mathrm{H}+\mathrm{Cl}^{-}$ & $0-2.5$ & Analogous to (109) & \\
\hline \multicolumn{5}{|c|}{ Penning and associative ionization, associative detachment } \\
\hline$(112)$ & $\mathrm{Ne}^{*}+\mathrm{Xe} \rightarrow \mathrm{Ne}+\mathrm{Xe}^{+}+\mathrm{e}$ & $7.5 \times 10^{-11}$ & Neynaber, Tang & [47] \\
\hline (113) & $\mathrm{Ne}^{*}+\mathrm{Xe} \rightarrow \mathrm{NeXe}^{+}+\mathrm{e}$ & $2.3 \times 10^{-11}$ & Neynaber, Tang & [47] \\
\hline (114) & $\mathrm{Cl}^{-}+\mathrm{H} \rightarrow \mathrm{HCl}(1)+\mathrm{e}$ & $6.0 \times 10^{-10}$ & Zwier et al. & [48] \\
\hline (115) & $\mathrm{Cl}^{-}+\mathrm{H} \rightarrow \mathrm{HCl}(2)+\mathrm{e}$ & $3.6 \times 10^{-10}$ & Zwier et al. & [48] \\
\hline \multicolumn{5}{|c|}{ Electron-ion recombination } \\
\hline$(116)$ & $\mathrm{NeXe}^{+}+\mathrm{e} \rightarrow \mathrm{Xe}^{*}+\mathrm{Ne}$ & $0.66 \times 10^{-7} / \sqrt{T_{\mathrm{e}}}$ & Hammer $\times 0.3$ & {$[10]$} \\
\hline (117) & $\mathrm{NeXe}^{+}+\mathrm{e} \rightarrow \mathrm{Xe}^{* *}+\mathrm{Ne}$ & $1.54 \times 10^{-7} / \sqrt{T_{\mathrm{e}}}$ & Hammer $\times 0.7$ & [10] \\
\hline (118) & $\mathrm{Xe}_{2}^{+}+\mathrm{e} \rightarrow \mathrm{Xe}^{*}+\mathrm{Xe}$ & $0.6 \times 10^{-7} / \sqrt{T_{\mathrm{e}}}$ & Bardsley, Biondi $\times 0.3$ & [49] \\
\hline (119) & $\mathrm{Xe}_{2}^{+}+\mathrm{e} \rightarrow \mathrm{Xe}^{* *}+\mathrm{Xe}$ & $1.4 \times 10^{-7} / \sqrt{T_{\mathrm{e}}}$ & Bardsley, Biondi $\times 0.7$ & [49] \\
\hline \multicolumn{5}{|c|}{ Dimer-ion formation } \\
\hline$(120)$ & $\mathrm{Ne}^{+}+\mathrm{Xe}+\mathrm{Ne} \rightarrow \mathrm{NeXe}^{+}+\mathrm{Ne}$ & $1.0 \times 10^{-31}$ & Levin et al. & {$[50]$} \\
\hline$(121)$ & $\mathrm{Xe}^{+}+\mathrm{Ne}+\mathrm{Ne} \rightarrow \mathrm{NeXe}^{+}+\mathrm{Ne}$ & $5.0 \times 10^{-32}$ & Kannari et al. & {$[51]$} \\
\hline (122) & $\mathrm{Xe}^{+}+\mathrm{Xe}+\mathrm{Ne} \rightarrow \mathrm{Xe}_{2}^{+}+\mathrm{Ne}$ & $1.0 \times 10^{-31}$ & Maeda et al. & [52] \\
\hline (123) & $\mathrm{Xe}^{+}+\mathrm{Xe}+\mathrm{Xe} \rightarrow \mathrm{Xe}_{2}^{+}+\mathrm{Ne}$ & $3.6 \times 10^{-31}$ & Gorse & [53] \\
\hline \multicolumn{5}{|c|}{ Charge exchange reactions } \\
\hline$(124)$ & $\mathrm{NeXe}^{+}+\mathrm{Ne} \rightarrow \mathrm{Xe}^{+}+\mathrm{Ne}+\mathrm{Ne}$ & $5.0 \times 10^{-10}$ & Kannari et al. & {$[51]$} \\
\hline (125) & $\mathrm{NeXe}^{+}+\mathrm{Xe} \rightarrow \mathrm{Xe}^{+}+\mathrm{Ne}+\mathrm{Xe}$ & $5.0 \times 10^{-10}$ & Levin et al. & {$[50]$} \\
\hline$(126)$ & $\mathrm{NeXe}^{+}+\mathrm{Xe} \rightarrow \mathrm{Xe}_{2}^{+}+\mathrm{Ne}$ & $5.0 \times 10^{-12}$ & Levin et al. & {$[50]$} \\
\hline$(127)$ & $\mathrm{Xe}_{2}^{+}+\mathrm{Ne} \rightarrow \mathrm{Xe}^{+}+\mathrm{Xe}+\mathrm{Ne}$ & $1.0 \times 10^{-12}$ & Estimted & \\
\hline (128) & $\mathrm{Cl}^{+}+\mathrm{Xe} \rightarrow \mathrm{Xe}^{+}+\mathrm{Cl}$ & $1.0 \times 10^{-12}$ & Levin et al. & {$[50]$} \\
\hline \multicolumn{5}{|c|}{ Ion-ion recombination (at standard discharge conditions) } \\
\hline$(129)$ & $\mathrm{Xe}^{+}+\mathrm{Cl}^{--} \rightarrow \mathrm{XeCl}^{*}(B)$ & $1.8 \times 10^{-6}$ & Flannery, Yang & [54] \\
\hline (130) & $\mathrm{Xe}_{2}^{+}+\mathrm{Cl}^{-} \rightarrow \mathrm{XeCl}^{*}(B)+\mathrm{Xe}$ & $1.8 \times 10^{-6}$ & Flannery, Yang & {$[54]$} \\
\hline (131) & $\mathrm{NeXe}^{+}+\mathrm{Cl}^{-} \rightarrow \mathrm{XeCl}^{*}(B)+\mathrm{Ne}$ & $1.8 \times 10^{-6}$ & Hokazono et al. & {$[55]$} \\
\hline (132) & $\mathrm{HCl}^{+}+\mathrm{Cl}^{-} \rightarrow \mathrm{Cl}_{2}^{*}+\mathrm{H}$ & $1.0 \times 10^{-6}$ & Kannari et al. & [51] \\
\hline$(133)$ & $\mathrm{Cl}^{+}+\mathrm{Cl}^{-} \rightarrow \mathrm{Cl}_{2}^{*}$ & $2.0 \times 10^{-6}$ & Levin et al. & {$[50]$} \\
\hline \multicolumn{5}{|c|}{ Neutral kinetics } \\
\hline$(134)$ & $\mathrm{Xe}^{*}+\mathrm{HCl}(0) \rightarrow \mathrm{Xe}+\mathrm{Cl}+\mathrm{H}$ & $5.6 \times 10^{-10}$ & Velazco et al. & {$[56]$} \\
\hline (135) & $\mathrm{Xe}^{*}+\mathrm{HCl}(1) \rightarrow \mathrm{XeCl}^{*}(B)+\mathrm{H}$ & $2.0 \times 10^{-10}$ & Chang & {$[57]$} \\
\hline (136) & $\mathrm{Xe}^{*}+\mathrm{HCl}(2) \rightarrow \mathrm{XeCl}^{*}(B)+\mathrm{H}$ & $2.0 \times 10^{-10}$ & Kannari et al. & {$[51]$} \\
\hline$(137)$ & $\mathrm{Xe}^{*}+\mathrm{HCl}(3) \rightarrow \mathrm{XeCl}^{*}(B)+\mathrm{H}$ & $2.0 \times 10^{-10}$ & Kannari et al. & {$[51]$} \\
\hline (138) & $\mathrm{Xe}^{* *}+\mathrm{HCl}(0) \rightarrow \mathrm{XeCl}^{*}(B)+\mathrm{H}$ & $4.2 \times 10^{-10}$ & $\mathrm{Ku}$, Setser & {$[58]$} \\
\hline (139) & $\mathrm{Xe} e^{* *}+\mathrm{HCl}(0) \rightarrow \mathrm{Xe}+\mathrm{Cl}+\mathrm{H}$ & $3.8 \times 10^{-10}$ & $\mathrm{Ku}$, Setser & {$[58]$} \\
\hline (140) & $\mathrm{Xe}^{* *}+\mathrm{HCl}(1) \rightarrow \mathrm{XeCl}^{*}(B)+\mathrm{H}$ & $8.0 \times 10^{-10}$ & Analogous to $(138,139)$ & \\
\hline (141) & $\mathrm{Xe}^{* *}+\mathrm{HCl}(2) \rightarrow \mathrm{XeCl}^{*}(B)+\mathrm{H}$ & $8.0 \times 10^{-10}$ & Analogous to $(140)$ & \\
\hline (142) & $\mathrm{Xe}^{* *}+\mathrm{HCl}(3) \rightarrow \mathrm{XeCl}^{*}(B)+\mathrm{H}$ & $8.0 \times 10^{-10}$ & Analogous to $(140)$ & \\
\hline (143) & $\mathrm{Cl}_{2}^{*}+\mathrm{Xe} \rightarrow \mathrm{XeCl}^{*}(B)+\mathrm{Cl}$ & $1.55 \times 10^{-10}$ & Analogous to $\mathrm{F}_{2}^{*}$ in Huestis et al. & [59] \\
\hline
\end{tabular}


Table 2 (continued)

\begin{tabular}{|c|c|c|c|c|}
\hline Number & Process & Energy range & Reference & \\
\hline $\begin{array}{l}(144) \\
(145) \\
(146) \\
(147) \\
(147) \\
(149) \\
(150) \\
(151)\end{array}$ & $\begin{array}{l}\mathrm{Ne}^{* *}+\mathrm{Ne} \rightarrow \mathrm{Ne}^{*}+\mathrm{Ne} \\
\mathrm{Xe}^{*}+\mathrm{Xe}+\mathrm{Ne} \rightarrow \mathrm{Xe}_{2}^{*}+\mathrm{Ne} \\
\mathrm{Xe}+\mathrm{Xe}+\mathrm{Xe} \rightarrow \mathrm{Xe}_{2}^{*}+\mathrm{Xe} \\
\mathrm{Xe}^{* *}+\mathrm{Ne} \rightarrow \mathrm{Xe}^{*}+\mathrm{Ne} \\
\mathrm{Xe}^{* *}+\mathrm{Xe} \rightarrow \mathrm{Xe}^{*}+\mathrm{Xe} \\
\mathrm{Cl}^{*}+\mathrm{Xe} \rightarrow \mathrm{Cl}+\mathrm{Xe}^{*} \\
\mathrm{Cl}^{*}+\mathrm{HCl}(0) \rightarrow \mathrm{Cl}_{2}^{*}+\mathrm{H} \\
\mathrm{H}+\mathrm{Cl}+\mathrm{Ne} \rightarrow \mathrm{HCl}(0)+\mathrm{Ne}\end{array}$ & $\begin{array}{l}3.0 \times 10^{-12} \\
1.6 \times 10^{-32} \\
8.0 \times 10^{-32} \\
2.5 \times 10^{-13} \\
1.1 \times 10^{-13} \\
1.0 \times 10^{-10} \\
5.0 \times 10^{-10} \\
1.1 \times 10^{-33}\end{array}$ & $\begin{array}{l}\text { Kannari et al. } \\
\text { Levin et al. } \\
\text { Hokazono et al. } \\
\text { Xu, Setser } \\
\text { Xu, Setser } \\
\text { Kannari et al. } \\
\text { Johnson et al. } \\
\text { Bruzzese }\end{array}$ & $\begin{array}{l}{[51]} \\
{[50]} \\
{[55]} \\
{[60]} \\
{[60]} \\
{[51]} \\
{[61]} \\
{[62]}\end{array}$ \\
\hline $\begin{array}{l}\text { Electron } \\
(152) \\
(153) \\
(154) \\
(155)\end{array}$ & $\begin{array}{l}\text { ching reactions } \\
\mathrm{XeCl}^{*}(B)+\mathrm{e} \rightarrow \mathrm{Xe}+\mathrm{Cl}+\mathrm{e} \\
\mathrm{XeCl}(X)+\mathrm{e} \rightarrow \mathrm{Xe}+\mathrm{Cl}+\mathrm{e} \\
\mathrm{Xe}_{2} \mathrm{Cl}^{*}+\mathrm{e} \rightarrow \mathrm{Xe}+\mathrm{Xe}+\mathrm{Cl}+\mathrm{e} \\
\mathrm{Cl}_{2}^{*}+\mathrm{e} \rightarrow \mathrm{Cl}+\mathrm{Cl}+\mathrm{e}\end{array}$ & $\begin{array}{l}3.2 \times 10^{-8} \\
7.0 \times 10^{-8} \\
2.0 \times 10^{-8} \\
2.0 \times 10^{-8}\end{array}$ & $\begin{array}{l}\text { Hammer } \\
\text { Wang } \\
\text { Johnson et al. } \\
\text { Johnson et al. }\end{array}$ & $\begin{array}{l}{[10]} \\
{[63]} \\
{[61]} \\
{[61]}\end{array}$ \\
\hline $\begin{array}{l}\text { Neutral q } \\
(156) \\
(157) \\
(158) \\
(159) \\
(160) \\
(161) \\
(162) \\
(163) \\
(164) \\
(165) \\
(166) \\
(167) \\
(168) \\
(169) \\
(170)\end{array}$ & 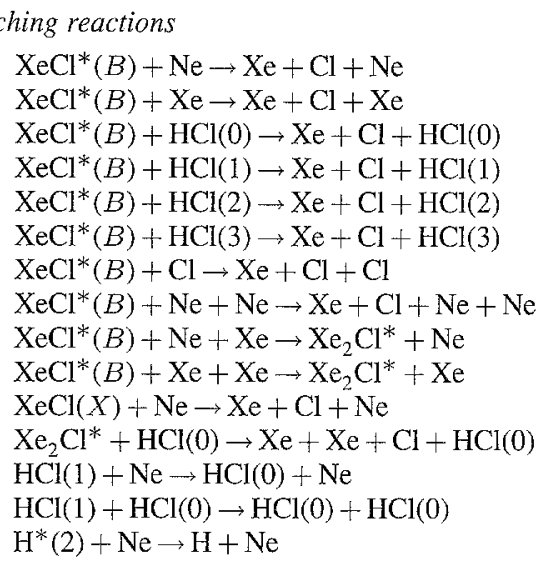 & $\begin{array}{l}7.6 \times 10^{-13} \\
5.0 \times 10^{-13} \\
7.7 \times 10^{-10} \\
7.7 \times 10^{-10} \\
7.7 \times 10^{-10} \\
7.7 \times 10^{-10} \\
8.0 \times 10^{-10} \\
1.0 \times 10^{-33} \\
6.0 \times 10^{-31} \\
1.3 \times 10^{-30} \\
3.04 \times 10^{-12} \\
8.0 \times 10^{-10} \\
6.2 \times 10^{-17} \\
2.7 \times 10^{-14} \\
1.0 \times 10^{-10}\end{array}$ & $\begin{array}{l}\text { Quiñones et al. } \\
\text { Quiñones et al. } \\
\text { Levin et al. } \\
\text { Levin et al. } \\
\text { Levin et al. } \\
\text { Levin et al. } \\
\text { Johnson et al. } \\
\text { Finn et al. } \\
\text { Quiñones et al. } \\
\text { Quiñones et al. } \\
\text { Hammer } \\
\text { Johnson et al. } \\
\text { Gorse } \\
\text { Gross, Bott } \\
\text { Estimated }\end{array}$ & $\begin{array}{l}{[64]} \\
{[64]} \\
{[50]} \\
{[50]} \\
{[50]} \\
{[50]} \\
{[61]} \\
{[65]} \\
{[64]} \\
{[64]} \\
{[10]} \\
{[61]} \\
{[53]} \\
{[66]}\end{array}$ \\
\hline $\begin{array}{l}\text { Spontaned } \\
(171) \\
(172) \\
(173) \\
(174) \\
(175) \\
(176) \\
(177) \\
(178) \\
(179) \\
(180) \\
(181) \\
(182)\end{array}$ & $\begin{array}{l}\text { emission } \\
\mathrm{XeCl}^{*}(B) \rightarrow \mathrm{XeCl}(X)+h \nu \\
\mathrm{Xe}_{2} \mathrm{Cl}^{*} \rightarrow \mathrm{Xe}+\mathrm{Xe}+\mathrm{Cl}+h \nu \\
\mathrm{Ne}^{* *} \rightarrow \mathrm{Ne}^{*}+h \nu \\
\mathrm{Xe}^{* *} \rightarrow \mathrm{Xe}^{*}+h \nu \\
\mathrm{Xe}_{2}^{*} \rightarrow \mathrm{Xe}+\mathrm{Xe}+h \nu \\
\mathrm{Cl}^{* *} \rightarrow \mathrm{Cl}+h \nu \\
\mathrm{Cl}_{2}^{*} \rightarrow \mathrm{Cl}+\mathrm{Cl}+h \nu \\
\mathrm{H}^{*}(3) \rightarrow \mathrm{H}^{*}(2)+h \nu \\
\mathrm{H}^{*}(4) \rightarrow \mathrm{H}^{*}(3)+h \nu \\
\mathrm{H}^{*}(5) \rightarrow \mathrm{H}^{*}(4)+h \nu \\
\mathrm{H}^{*}(6) \rightarrow \mathrm{H}^{*}(5)+h \nu \\
\mathrm{H}^{*}(7) \rightarrow \mathrm{H}^{*}(6)+h \nu\end{array}$ & $\begin{array}{l}9.09 \times 10^{7} \\
7.4 \times 10^{6} \\
4.7 \times 10^{7} \\
3.62 \times 10^{7} \\
6.0 \times 10^{7} \\
2.15 \times 10^{7} \\
1.0 \times 10^{8} \\
4.41 \times 10^{7} \\
8.99 \times 10^{6} \\
2.70 \times 10^{6} \\
1.03 \times 10^{6} \\
4.56 \times 10^{5}\end{array}$ & $\begin{array}{l}\text { Hay, Dunning } \\
\text { Johnson et al. } \\
\text { Wiese et al. } \\
\text { Aymar, Coulombe } \\
\text { Levin et al. } \\
\text { Wiese et al. } \\
\text { Diegelmann et al. } \\
\text { Wiese et al. } \\
\text { Wiese et al. } \\
\text { Wiese et al. } \\
\text { Wiese et al. } \\
\text { Wiese et al. }\end{array}$ & $\begin{array}{l}{[67]} \\
{[61]} \\
{[68]} \\
{[69]} \\
{[50]} \\
{[71]} \\
{[70]} \\
{[68]} \\
{[68]} \\
{[68]} \\
{[68]} \\
{[68]}\end{array}$ \\
\hline
\end{tabular}

states will not influence the ionization kinetics significantly. During the Townsend phase (Sect. 3.1) the effective ionization depends on the total excitation and the direct ionization cross sections. The electron collision excitation cross sections of the lower excited states ( $\mathrm{Xe}^{*}$ and $\mathrm{Ne}^{*}$ ) result from the sum of the $(n+1) s$ and $(n+1) s^{\prime}$ excitation cross sections obtained by Puech and Mizzi [30]. In order to take into account a correct power loss due to electron-collision excitation of xenon and neon and thus the sensitivity of the Townsend ionization coefficient to the excitation, the cross sections of the upper excited states $\left(\mathrm{Xe}^{* *}\right.$ and $\mathrm{Ne}^{* *}$ ) are determined by substracting the cross sections of the lower states from the total excitation cross sections of Puech and Mizzi [30]. In our new kinetic model the ionization cross sections of xenon and neon given by Rapp and EnglanderGolden [72] have been replaced by the cross sections recently measured by Wetzel et al. [41].
When modelling XeCl-laser plasmas, the reactions of electrons with $\mathrm{HCl}$ are very important. In our kinetic model we include five vibrationally excited states of $\mathrm{HCl}$ in addition to the ground state. The electron collision vibrational excitation of $\mathrm{HCl}(v)$ with $v=0-5$ has been improved using theoretically calculated cross sections by Domcke and Mündel [33] and by Domcke [34]. The latter cross-section data [34] have been calculated with the same model [33] and reduce the uncertainties in the stepwise $\mathrm{HCl}$ vibrational excitation kinetics of the previous kinetic model [13, 14]. Furthermore, electronic excitations and ionization of $\mathrm{HCl}$ have been added to the model. We use the electron-collision cross sections given by Davies [35] with the excitation to the $\mathrm{HCl}^{*}(A)$ state being treated as direct dissociation. The cross sections for dissociative attachment of electrons by $\operatorname{HCl}(v)$ with $v=0,1,2$ have been taken from Domcke and Mündel [33], while those for $v>2$ have been put equal to 
$\mathrm{HCl}(v=2)$ for energies higher than $0.4 \mathrm{eV}$ in combination with the cross sections calculated by Bardsley and Wadehra [46] for $\mathrm{HCl}(v=3)$. The dissociative electron attachment of $\mathrm{HCl}(v=0)$ resulting in $\mathrm{H}^{-}$and $\mathrm{Cl}$ has as well been taken into account by use of the cross sections obtained by Davies [35].

The electron kinetics is predominantly determined by direct and multistep ionization of $\mathrm{Xe}$ and dissociative electron attachment of $\mathrm{HCl}$. The electron collision processes of chlorine and hydrogen and their excited states can be neglected with regard to the ionization and recombination kinetics. A detailed discussion of the electron collision cross sections used for chlorine and hydrogen will be given in a subsequent paper [15] dealing with the comparison of calculated and spectroscopically measured number densities of various heavy-particle components. A complete list of the numerical values actually used is given in [73] and is available on request.

The ionization kinetics of the excited states of xenon is strongly influenced by the quenching reactions $\mathrm{Xe}^{* *}+\mathrm{M} \rightarrow \mathrm{Xe}^{*}+\mathrm{M}$ with $\mathrm{M}=\mathrm{Ne}$, Xe. Since the quenching of $\mathrm{Xe}\left(6 p(1 / 2)_{1}\right)$ largely governs the transfer rate of the $\mathrm{Xe}^{* *}$ population to the $\mathrm{Xe}^{*}$ levels, especially to $\operatorname{Xe}\left(6 s^{\prime}(1 / 2)_{1}\right)$, the rate coefficients for the reactions (147) and (148) have been deduced from [60] by statistically weighting with a factor of $g\left[\mathrm{Xe}\left(6 p(1 / 2)_{1}\right] / g\left(\mathrm{Xe}^{* *}\right)=1 / 12\right.$.

In order to give a correct description of the electron kinetics at low $\mathrm{HCl}$ pressures we included the molecular ions $\mathrm{Xe}_{2}^{+}$and $\mathrm{NeXe}^{+}$in our model. The influence of the $\mathrm{NeXe}^{+}$ion will be discussed.

\subsection{Model of the Electrical Circuit}

Due to their capability of temporarily storing and delivering energy, inductances and capacities distributed within the discharge chamber and the high-voltage feedthrough may influence the discharge voltage especially during the ignition phase (see Sect. 3.1) when discharge voltage and current are changing rapidly. Therefore, a correct model of the discharge has to include an equivalent electrical circuit of the discharge chamber. The equivalent electrical circuit and the according numerical values are given in Fig. 1. Slight changes in $C_{\text {Dis }}$ and $L_{2}$ with respect to [7] are due to minor changes of the electrode geometry. The comparison of the

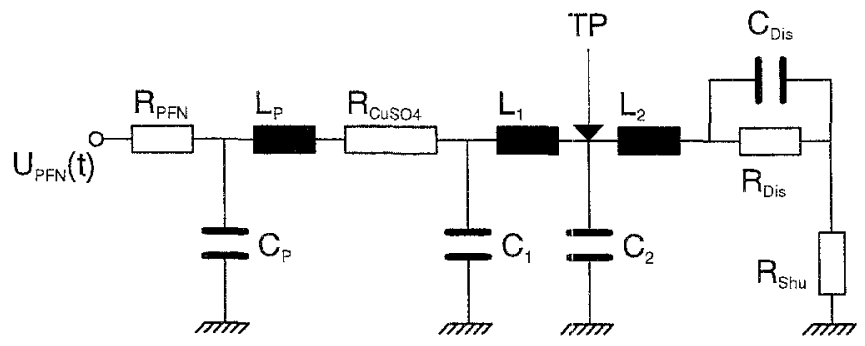

Fig. 1. Equivalent electrical circuit of the discharge chamber. $C_{\mathrm{p}}=$ $8 \mathrm{pF}, C_{1}=21 \mathrm{pF}, C_{2}=3 \mathrm{pF}, C_{\text {Dis }}=2.6 \mathrm{pF}$ (at standard conditions), $L_{\mathrm{p}}=70 \mathrm{nH}, L_{1}=15 \mathrm{nH}, L_{2}=17 \mathrm{nH}, R_{\mathrm{PFN}}=50 \Omega, R_{\mathrm{CuSo}_{4}}=48 \Omega$, $R_{\text {Shu }}=83 \mathrm{~m} \Omega, U_{\mathrm{PFN}}(t)=$ voltage of the pulse generator. TP marks the point where the voltage is measured. $L_{2} \mathrm{~d} I / \mathrm{d} t$ is a small correction which is taken into account

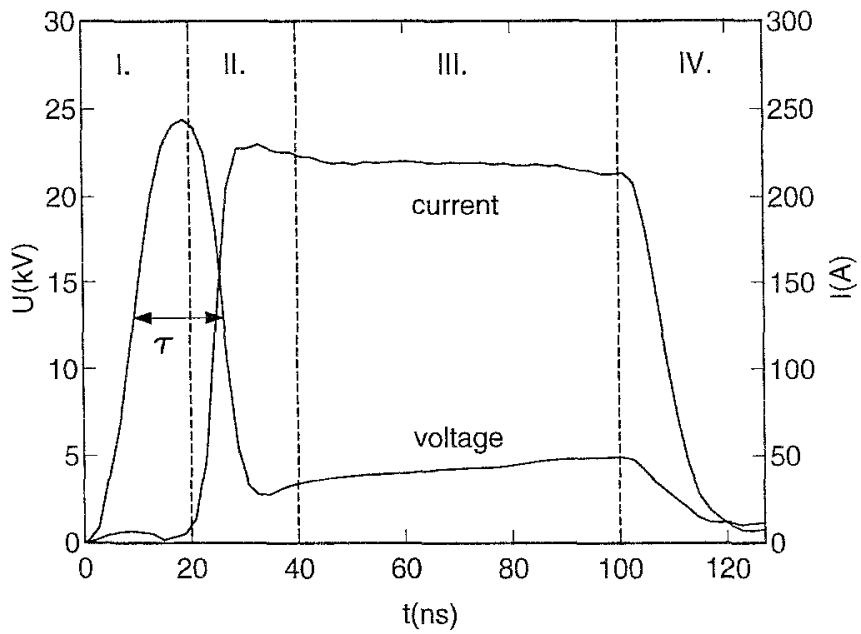

Fig. 2. Discharge voltage and current at standard discharge conditions. The different discharge phases defined in Sect. 3.1 are indicated

measured response to a fast rising high-voltage pulse with the calculated one based on this equivalent circuit shows good agreement.

\section{Results and Discussion}

\subsection{Discharge Voltages}

Voltage measurements were done for a large variety of discharge parameters (see Table 3). Figure 2 shows the current and voltage traces at standard discharge conditions.

According to the changes in the dominating reaction kinetics, we will define four different discharge phases (as indicated in Fig. 2). The Townsend phase is the beginning of the discharge evolution when space charges in the cathode region are unimportant and when all the processes can be described by $E / N$-dependent rate coefficients as the number densities of secondary species generated during the temporal evolution of the discharge are still small. This phase ends when the discharge current density reaches some $\mathrm{A} / \mathrm{cm}^{2}$. During the ignition phase the discharge voltage falls off rapidly because the fast rising current causes a large voltage drop at the impedance of the pulse generator and the current-limiting resistor. The Quasi-Steady-State (QSS) phase begins with the stabilization of the discharge voltage and current and ends with the applied voltage pulse when the recombination phase begins.

Table 3. Discharge parameters for which voltage measurements were done

\begin{tabular}{ll}
\hline Quantities changed & Parameter range \\
\hline $\mathrm{HCl}$ partial pressure & $0-10 \mathrm{mbar}$ \\
Charging voltage $U_{\mathrm{Ch}}$ & $12.5-30 \mathrm{kV}$ \\
Xe partial pressure & $50-1000 \mathrm{mbar}$ \\
Neon buffer gas pressure & $0.3-10 \mathrm{bar}$ \\
Discharge cross section & circular, $5-10 \mathrm{~mm}$ \\
Preionization electron density & $10^{8}-1.3 \times 10^{9} / \mathrm{cm}^{3}$ \\
He and Ar as buffer gases & $1-3 \mathrm{bar}$ \\
\hline
\end{tabular}




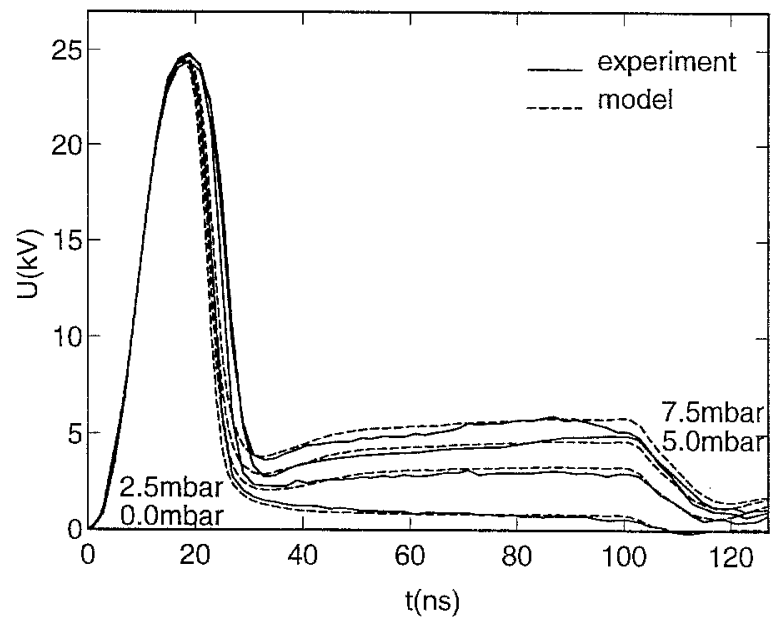

Fig. 3. Discharge voltage at different partial pressures of HCl. $P_{\mathrm{Xe}}=$ 75 mbar, total gas pressure $P=3 \mathrm{bar}, U_{\mathrm{Ch}}=25.9 \mathrm{kV}$

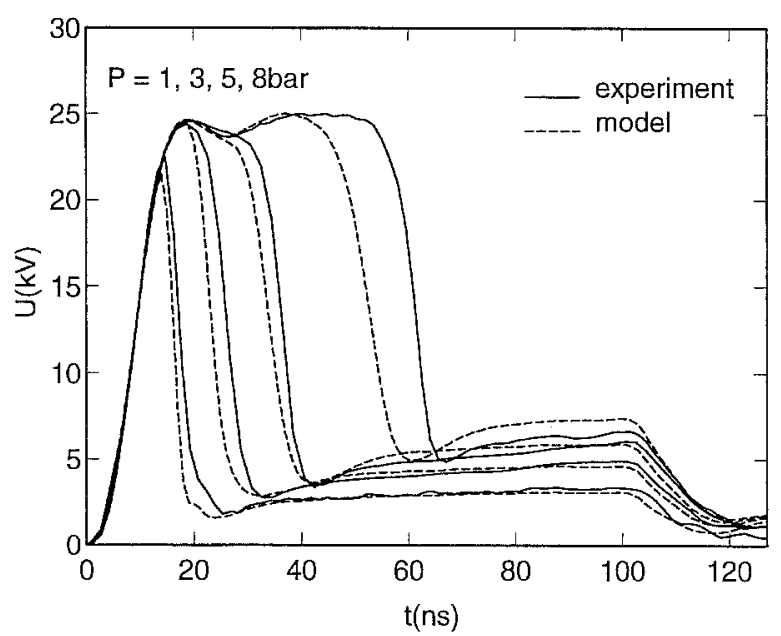

Fig. 4. Discharge voltage at different buffer gas pressures. $P_{\mathrm{HCl}}=$ 5 mbar, $P_{\mathrm{Xe}}=75 \mathrm{mbar}, U_{\mathrm{Ch}}=25.9 \mathrm{kV}$

The most sensitive check of the reaction kinetics used in the model calculations is given by the discharge voltage $U_{\text {Dis }}$. Characteristic data to be compared are the breakdown delay time $\tau$ (Fig. 2) and the discharge voltage during the QSS phase.

As current and voltage are correlated via the impedance of the pulse-forming network and the current-limiting resistor [7], we will only display the voltage traces in the following figures.

The comparison of the discharge voltages of experiment and numerical model for different $\mathrm{HCl}$ contents, buffer gas pressures, and applied voltages (and therefore different current densities as well) is shown in Figs. 3, 4, and 5, respectively. The measured voltages are well reproduced by the model calculations during the QSS phase whereas there is a significant difference in the breakdown delay times.

During the Townsend phase the electron production is predominated by the direct ionization of $\mathrm{Xe}$, while the main electron loss is by dissociative electron attachment of $\mathrm{HCl}$ molecules in the vibrational ground state $\operatorname{HCl}(v=0)$. As the discharge voltage and thus the electrical field rises, the ionization very soon exceeds the attachment and the electron density increases. When the electron density and, therefore, the current have become large enough, the voltage falls off.

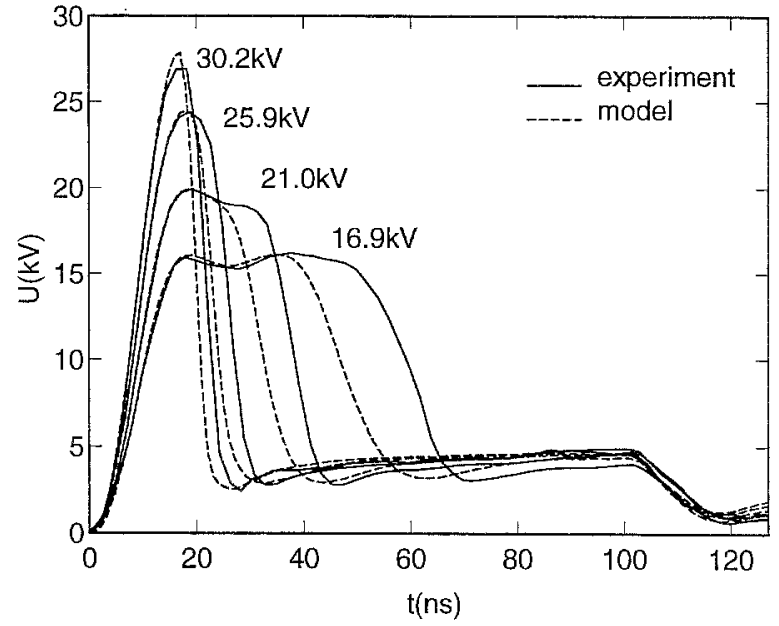

Fig. 5. Discharge voltage at different charging voltages $U_{\mathrm{Ch}}$. $P_{\mathrm{HCl}}=$ 5 mbar, $P_{\mathrm{Xe}}=75 \mathrm{mbar}, P_{\mathrm{Ne}}=2920 \mathrm{mbar}$

Due to the rising electron density, the number densities of the excited states of xenon atoms and of the vibrationally excited $\mathrm{HCl}$ molecules $\mathrm{HCl}(v>0)$ increase causing a change in the ionization and recombination kinetics and thereby defining the beginning of the ignition phase. From now on the ionization of $\mathrm{Xe}^{* *}$ and, to a lesser extent, that of $\mathrm{Xe}^{*}$ become the leading processes for the electron production. At the beginning of the ignition phase $\operatorname{HCl}(v=0)$ still dominates the electron loss since $\mathrm{HCl}(v>0)$ is not yet significantly populated. With the increasing number densities of $\mathrm{HCl}(v>0)$ the dissociative electron attachment increases as well and the discharge voltage rises. The precise timing of the counteracting increasing ionization processes and more slowly increasing attachment (and recombination) processes in the model shows itself in the reproduction of the voltage minimum.

During the QSS phase ionization and recombination nearly balance each other. The electron loss is dominatecl by $\operatorname{HCl}(v=2)$ and $\operatorname{HCl}(v=1)$, while the influence of $\mathrm{HCl}(v=3)$ slowly increases during the QSS phase and becomes important in the late QSS phase only. In contrast to $[12,74]$ we found $\mathrm{HCl}(v>3)$ to have no significant influence on the reaction kinetics as the voltage pulses of our experimental setup have a duration of less than $100 \mathrm{~ns}$. This result holds even if the electronic excitation of $\mathrm{HCl}(v>0)$ to $\operatorname{HCl}^{*}(A)$ and $\operatorname{HCl}^{*}(B, C)$ with the electron collision cross sections derived in accordance with Longo et al. [75], the vibration-translation processes of $\operatorname{HCl}(v \geq 1)$ with $\mathrm{H}$, $\mathrm{Cl}$, and $\mathrm{HCl}(0)$, and the vibration-vibration processes of $\mathrm{HCl}(v \geq 1)$ are taken into account, using the rate coefficients reported in [66] for the latter.

In agreement with $[12,76]$ and in contrast to $[9,77]$, our investigations show that $\mathrm{NeXe}^{+}$(as well as $\mathrm{Xe}_{2}^{+}$) is of minor importance in discharges containing $\mathrm{HCl}$. In $\mathrm{HCl}$-free discharges the ionization is balanced by electron recombination with these molecular ions.

The proper choice of the quenching rate coefficients for $\mathrm{Xe}^{* *}+\mathrm{M} \rightarrow \mathrm{Xe}^{*}+\mathrm{M}$ with $\mathrm{M}=\mathrm{Ne}$, Xe is important for the description of the ionization kinetics. The reproduction of the voltage minimum and the discharge voltage during the QSS phase for the various discharge conditions could only be achieved with the rate coefficients used in our kinetic model. 


\subsection{Breakdown Delay Times}

The lack in the reproduction of the measured breakdown delay times by the model cannot be explained by experimental inaccuracies in the measurement of the partial gas pressures, the gas temperature, the discharge voltage, the electrode gap, the preionization electron density, the discharge cross section, or the gas pollution. The electron-electron interaction as well as superelastic collisions where found to have no significant influence on the breakdown delay times because the electron density and the densities of the excited atoms and molecules are too small during the Townsend phase and at the beginning of the ignition phase.

The discrepancy in the breakdown delay times can be explained in two different ways.

1. An assumed cathode fall would reduce the electrical field and the ionization frequency in the bulk plasma and thus enhance the breakdown delay time. A constant cathode-fall voltage during the whole Townsend phase and the beginning of the ignition phase of some hundred volts according to the limits of the cathode-fall voltage measured at the beginning of the QSS phase (see Sect. 1.2) does not reproduce the measured breakdown delays. Agreement between experiment and model can be achieved by increasing the assumed cathode-fall voltage to about $3 \mathrm{kV}$. Such a cathode-fall voltage would lead to a significantly prolonged breakdown delay time when the electrode gap is reduced, which could not be observed experimentally. Additionally, it is evident that during the Townsend phase (as the name says) the cathode fall has not yet developed because the space-charge density in the cathode region is still too small. We can therefore conclude that the inconsistency in the breakdown delay times is not due to a constant cathode fall. Cathode-fall voltages of the order of $10^{4} \mathrm{~V}$ that exist for a short time of about $3 \mathrm{~ns}$, i.e. only during a part of the ignition phase, would explain the discrepancy between experiment and model and do not contradict the experimentally observed fact that the breakdown delay time remains constant, when the electrode gap and the applied voltage, accordingly, are reduced.

2. In the kinetic model the manifold of the Xe levels is represented by the ground state (Xe), two excited states $\left(\mathrm{Xe}^{*}, \mathrm{Xe}^{* *}\right)$, and the ion $\left(\mathrm{Xe}^{+}\right)$, as described in Sect. 2.2 . An increase of the electron excitation of $\mathrm{Xe}(\mathrm{Xe}+\mathrm{e} \rightarrow$ $\mathrm{Xe}^{*}+\mathrm{e}$ and $\mathrm{Xe}+\mathrm{e} \rightarrow \mathrm{Xe}^{* *}+\mathrm{e}$ with threshold energies of $8.32 \mathrm{eV}$ and $9.58 \mathrm{eV}$, respectively) leads to a diminution of the high-energy part of the electron energy-distribution function as these electrons are consumed by the excitation of Xe. Therefore, the amount of electrons available for direct ionization of Xe will decrease, if the electron excitation cross sections ( $\mathrm{Xe}+\mathrm{e} \rightarrow \mathrm{Xe}^{*, * *}+\mathrm{e}$ ) are enhanced. Enlarging the cross sections by about $25 \%$ above the values given in literature [30], which within the experimental error bounds, reduces the ionization frequency and thereby increases the breakdown delay time by the right amount to obtain agreement between experimental and theoretical voltage traces (Figs. 6,7). At the same time it do not influence the good reproduction of the discharge voltage during the QSS phase. This agreement has shown to be valid within a wide range of discharge parameters not only in $\mathrm{Ne} / \mathrm{Xe} / \mathrm{HCl}$ discharges, but in $\mathrm{He} / \mathrm{Xe} / \mathrm{HCl}$ discharges as well.

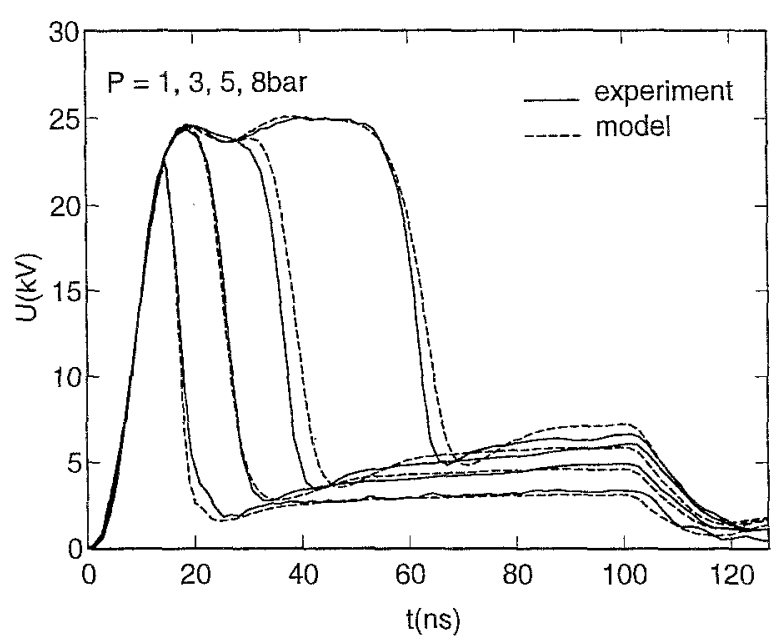

Fig. 6. Discharge voltage at different buffer gas pressures with enhanced excitation cross sections for the model calculations. Discharge parameters as in Fig. 4

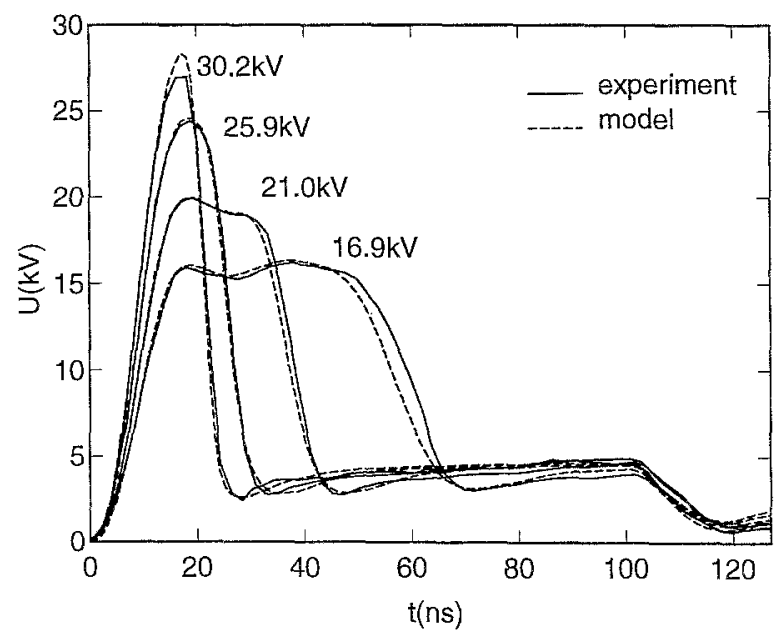

Fig. 7. Discharge voltage at different charging voltages $U_{\mathrm{Ch}}$ with enhanced excitation cross sections for the model calculations. Discharge parameters as in Fig. 5

As two possible causes can account for the observed breakdown delay times, further experiments will have to show in how far each of the two contributes to the overall effect.

\section{Conclusions}

A new 0-dimensional model of transient high-pressure glow discharges has been developed. Its essential features are an improved code solving the nonstationary electron Boltzmann equation with an energy grid which has 1000 grid points and an updated set of reactions and electron-collision cross sections using the most recent literature. The predictions of the model have been checked by measuring the voltage drop in a special, very homogeneous discharge. From the experiment an upper limit of the cathode-fall voltage in the ignited discharge has been deduced. Using slightly enhanced cross sections for the total electron-collision excitation of xenon we could reach excellent agreement of experiment and model in a large field of parameters. This enhancement is necessary to reproduce the measured breakdown delay times. The necessary increase of the breakdown delay times 
of the model can also be explained by the assumption of a very short $(\sim 3 \mathrm{~ns})$, very high $(\sim 10 \mathrm{kV})$ transient cathode fall during the ignition of the discharge. We recommend to use the reaction kinetics with the enhanced cross sections as a reference kinetics when deriving simplified kinetics for multidimensional models.

Acknowledgements. We would like to thank R. Winkler and Th. Hammer for many helpful discussions and suggestions during the model development and $\mathrm{W}$. Domcke for providing us with the $\mathrm{HCl}$ cross sections. The experiments, especially the optical investigations concerning the discharge homogeneity, benefited from the close cooperation with R. Dreiskemper, (I. Schröder, and A. Schwabedissen. The model development was in part sponsored by the BMFT R \& D projects 13 N5722/3 and $13 \mathrm{~N} 5406 / 9$.

\section{References}

1. M.J. Kushner, A.L. Pindroh, C.H. Fisher, T.A. Znotins, J.J. Ewing: J. Appl. Phys. 57, 2406 (1985)

2. M. Bähr: Untersuchungen zur Stabilität von $\mathrm{XeCl}^{*}$-Entladungen, Dissertation, Universität Hannover (1989)

3. R. Sorkina: J. Phys. D 23, 806 (1990)

4. M. Bähr, W. Bötticher, S. Choroba: IEEE Trans. PS-19, 369 (1991)

5. A. Belasri, S. Longo, C. Gorse, M. Capitelli, J.P. Boeuf, L.C. Pitchford: XXth ICPIG, Barga, Contr. Papers (1991) pp. 1170

6. M.M. Turner: J. Appl. Phys. 71, 2113 (1992)

7. W. Bötticher, H. Lück, S. Niesner, A. Schwabedissen: Appl. Phys. B 54, 295 (1992)

8. D. Loffhagen, R. Winkler: J. Comp. Phys. (in press)

9. R. Winkler, M.W. Wuttke: Appl. Phys. B 54, 1 (1992)

10. T. Hammer: Excimer Lasers and Applications II, Proc. SPIE 1278, 119 (1990)

11. C. Gorse, M. Capitelli, A. Dipace: J. Appl. Phys. 67, 1118 (1990)

12. E. Estocq, G. Delouya, J. Bretagne: Appl. Phys. B 56, 209 (1993)

13. G. Stielow, Th. Hammer, W. Bötticher: Appl. Phys. B 47, 333 (1988)

14. Th. Hammer, W. Bötticher: Appl. Phys. B 48, 73 (1989)

15. A. Schwabedissen, D. Loffhagen, Th. Hammer, W. Bötticher: Appl. Phys. B (submitted)

16. H. Lück: Präzisionsmessung von Entladungsstrom und -spannung von Hochdruckglimmentladungen in $\mathrm{Ne}: \mathrm{Xe}: \mathrm{HCl}$ Gemischen. Dissertation, Universität Hannover (1992)

17. M.C. Cavenor, J. Meyer: Aust. J. Phys. 22155 (1969)

18. A. Belasri, J.P. Boeuf, L.C. Pitchford: J. Appl. Phys. 74, 1553 (1993)

19. T. Steihaug, A. Wolfbrandt: Math. Comp. 33, 521 (1979)

20. R.E. Scraton: Int. J. Comp. Math. 9, 81 (1981)

21. T.F. O'Malley, R.W. Crompton: J. Phys. B 13, 3451 (1980)

22. W.C. Fon, K.A. Berrington: J. Phys. B 14, 323 (1981)

23. L.T. Sin Fai Lam: J. Phys. B 15, 119 (1982)

24. M. Hayashi: J. Phys. D 16, 581 (1983)

25. N.T. Padial, D.W. Norcross, L.A. Collins: Phys. Rev. A 27, 141 (1983)

26. T. Hammer: Untersuchungen zur Reaktionskinetik gasentladungsgepumpter $\mathrm{XeCl}^{*}$-Laser mittels hochzeitaufgelöster absorptionsspektroskopischer Messungen von Xe*-Teilchenzahldichten. Dissertation, Universität Hannover (1987)

27. G.L. Rogoff, J.M. Kramer, R.B. Piejak: IEEE Trans. PS-14, 103 (1986)

28. W.C. Fon, P.G. Burke, A.E. Kingston: J. Phys. B 11, 521 (1978)

29. W.C. Fon, K.A. Berrington, P.G. Burke, A.E. Kingston: J. Phys. B 14, 1041 (1981)

30. V. Puech, S. Mizzi: J. Phys. D 24, 1974 (1991)

31. M.J. Seaton: Proc. Phys. Soc. 79, 1105 (1962)

32. H.A. Hyman: Phys. Rev. A 24, 1094 (1981)

33. W. Domcke, C. Mündel: J. Phys. B 18, 4491 (1985)

34. W. Domcke: Private communication
35. D.K. Davies: Westinghouse Report AFWAL-TR-82-2083 (1982)

36. P.S. Ganas: J. Appl. Phys. 63, 277 (1988)

37. J. Callaway: Phys. Rev. A 26, 199 (1982)

38. J. Callaway: Phys. Rev. A 32, 775 (1985)

39. J. Callaway, K. Unnikrishnan, D.H. Oza: Phys. Rev. A 36, 2576 (1987)

40. L. Vriens, A.H.M. Smeets: Phys. Rev. A 22, 940 (1980)

41. R.C. Wetzel, F.A. Baiocchi, T.R. Hayes, R.S. Freund: Phys. Rev. A 35, 559 (1987)

42. H.A. Hyman: Phys. Rev. A. 20, 855 (1979)

43. T.R. Hayes, R.C. Wetzel, R.S. Freund: Phys. Rev. A 35, 578 (1987)

44. M.B. Shah, D.S. Elliot, H.B. Cilbody: J. Phys. B 20, 3501 (1987)

45. H.S.W. Massey: Proc. Soc. 155, 472 (1936)

46. J.N. Bardsley, J.M. Wadehra: J. Chem. Phys. 78, 7227 (1983)

47. R.H. Neynaber, S.Y. Tang: J. Chem. Phys. 70, 4272 (1979)

48. T.S. Zwier, M.M. Maricq, C.J.S. M. Simpson, V.M. Bierbaum, C.B. Ellison, S.R. Leone: Phys. Rev. Lett. 44, 1050 (1980)

49. J.N. Bardsley, M.A. Biondi: Adv. At. Mol. Phys. 6, 2 (1970)

50. L.A. Levin, S.E. Moody, E.L. Klostermann, R.E. Center, J.J. Ewing: IEEE J. QE-17, 2282 (1981)

51. F. Kannari, W.D. Kimura, J.J. Ewing: J. Appl. Phys. 68, 2615 (1990)

52. M. Maeda, A. Takahashi, T. Mizunami, Y. Miyazoe: Jpn. J. Appl. Phys. 21, 1161 (1982)

53. C. Gorse: In Nonequilibrium Processes in Partially Ionized Gases, ed. by M. Capitelli, J.N. Bardsley (Plenum, New York 1990) pp. 411

54. M.R. Flannery, T.P. Yang: Appl. Phys. Lett. 32, 327 (1978)

55. H. Hokazono, K. Midorikawa, M. Obara, T. Fujioka: J. Appl. Phys. 56, $680(1984)$

56. J.E. Velazco, J.H. Kolts, D.W. Setser: J. Chem. Phys. 69, 4357 (1978)

57. R.S.F. Chang: J. Chem. Phys. 76, 2943 (1982)

58. J.K. Ku, D.W. Setser: Appl. Phys. Lett. 48, 689 (1986)

59. D.L. Huestis, R.M. Hill, H.H. Nakano, D.C. Lorents: J. Chem. Phys. 69, 5133 (1978)

60. J. Xu, D.W. Setser: J. Chem. Phys. 94, 4243 (1991)

61. T.H. Johnson, H.E. Cartland, T.C. Genoni, A.M. Hunter: J. Appl. Phys. 66, 5707 (1989)

62. R. Bruzzese: Lett. Nuovo Cimento 40, 45 (1984)

63. Y.-.S. Wang: A Theorectical Study of the Kinetic Processes in a High-Power Xenon Chloride Excimer Laser Oscillator Driven by a Transmission Line Pulse Forming Network. Ph. D. Thesis, University of California, San Diego (1982)

64. E. Quiñones, Y.C. Yu, D.W. Setser, G. Lo: J. Chem. Phys. 93, 333 (1990)

65. T.G. Finn, R.S.F. Chang, L.J. Palumbo, L.F. Champagne: Appl. Phys. Lett. 36, 789 (1980)

66. R.W.F. Gross, J.F. Bott: Handbook of Chemical Lasers (Wiley, New York 1976)

67. P.J. Hay, T.H. Dunning: J. Chem. Phys. 69, 2209 (1978)

68. W.L. Wiese, M.W. Smith, B.M. Glennon: Atomic Transition Probabilities, Vol. I (NSRDS-NBS 4, Washington, DC 1966)

69. M. Aymar, M. Coulombe: At. Data Nucl. Data Tables 21, 537 (1978)

70. M. Diegelmann, K. Hohla, F. Rebentrost, K.L. Kompa: J. Chem. Phys. 76, 1233 (1982)

71. W.L. Wiese, M.W. Smith, B.M. Glennon: Atomic Transition Probabilities, Vol. II (NSRDS-NBS 22, Washington DC 1969)

72. D. Rapp, P. Englander-Golden: J. Chem. Phys. 43, 1464 (1965)

73. D. Backhaus: Untersuchungen zur instationären Boltzmanngleichung und zur Reaktionskinetik in der Modellierung von $\mathrm{XeCl}^{*}$ und $\mathrm{KrF}^{*}$-Hochdruckglimmentladungen. Dissertation, Universität Hannover (1992)

74. C. Corse, M. Capitelli, S. Longo, E. Estocq, J. Bretagne: J. Phys. D 24, 1947 (1991)

75. S. Longo, M. Capitelli, C. Gorse, A.V. Dem'janov, I.V. Kochetov, A.P. Napartovich: Appl. Phys. B 54, 239 (1992)

76. R. Sorkina, F.A. van Goor, W.J. Witteman: Appl. Phys. B 55, 478 (1992)

77. S. Longo, C. Gorse, M. Capitelli: IEEE Trans. PS-19, 379 (1991) 